Chinese Journal of

Astronomy and

Astrophysics

\title{
Polarization in Gamma-Ray Bursts Produced by Pinch Discharge
}

\author{
Mei $\mathrm{Wu}^{1}, \mathrm{Li} \mathrm{Chen}^{2}$ and Ti-Pei $\mathrm{Li}^{1,3}$ \\ ${ }^{1}$ Key Lab. of Particle Astrophys., Inst. of High Energy Phys., Chinese Academy of Sciences \\ 2 Department of Astronomy, Beijing Normal University \\ ${ }^{3}$ Center for Astrophysics, Tsinghua University, Beijing
}

\begin{abstract}
Large-voltage and high-temperature plasma columns produced by pinch discharge can generate $\gamma$-ray flashes with energy spectra and spectral evolution consistent with that observed in $\gamma$-ray bursts (GRBs). The inverse Compton scattering (ICS) during the discharge process can produce high linear polarization. The calculation indicates that the observed polarization depends on the angle between the observer's line-of-sight to the GRB and the direction of the pinch discharge, but only weakly depends on observed $\gamma$-ray energy.
\end{abstract}

Key words: gamma-ray: bursts — radiation mechanism: non thermal — polarization

\section{INTRODUCTION}

Coburn and Boggs (2003) reported their analysis of GRB 021206 observed by the Reuven Ramaty High Energy Solar Spectroscopic Imager (RHESSI). They found that a high degree, $(80 \pm 20) \%$, of linear polarization is present in the prompt emission in $25-2000 \mathrm{keV}$. But, due to the limited ratio of signal to noise of the data, Rutledge \& Fox (2004) concluded a null polarization and Wigger et al. (2004) obtained the linear polarization degree of $\left(41_{-44}^{+57}\right) \%$ from the same data. The study of polarization is important in understanding the production mechanism of GRBs, though the detection is still uncertain at the present stage. It is expected that more reliable measurements in the future for GRB polarization may give strong constraint on different GRB models.

Based on the fireball model, some theoretical considerations have been done to explain the possible polarization. Lyutikov et al. (2003) believed that the large scale ordered magnetic fields produced at the central source may produce highly polarized GRB prompt emission. The calculation demanded the magnetic field in the emission region is dominated by the toroidal field and is concentrated in a thin shell near the surface of the shell expanding with the Lorentz factor and the synchrotron emission is produced by an isotropic population of relativistic electrons with the power law distribution in energy. Strong polarization might arise in a jet with the line-of-sight to the GRB being close to the jet edge (Medvedev \& Loeb 1999; Gruzinov 1999;

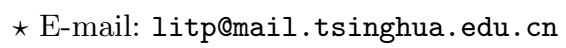




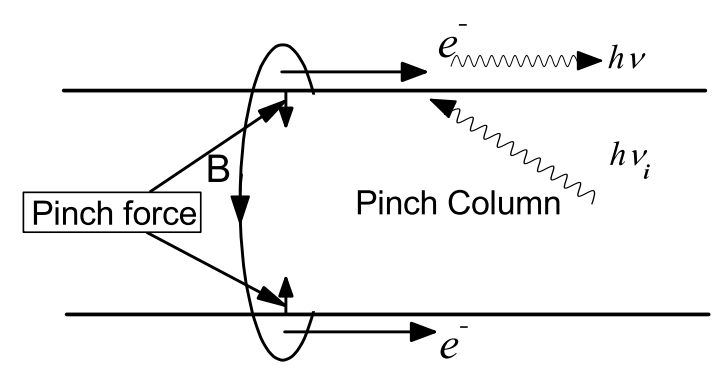

Fig. 1 The pinch discharge model of GRBs.

Waxman 2003). In this case, the polarization signal may be not averaged out. Dar \& De Rujula (2003) and Eichler \& Levinson (2003) discussed polarization as a characteristic signature of inverse Compton up-scatter. Lazzati et al. (2003) considered Compton drag as a mechanism for high linear polarization. In such scenario, the relativistic electrons are contained in a fireball, and the shape of the soft photon field is selected in order to reproduce the GRB spectra.

We have proposed an electrical discharge model of GRBs (Li \& Wu 1997): large-voltage and high-temperature pinch plasma columns produced by disruptive electrical discharges can generate $\gamma$-ray flashes with energy spectra and spectral evolution characters consistent with that observed in GRBs. In this paper we show that the pinch discharge process can naturally produce the high degree polarization.

\section{DISCHARGE MODEL}

Alfvén (1981) stressed the importance of electric currents in plasmas to understanding phenomena occurred in the magnetosphere till galactic dimensions. A current flowing in the plasma may contract by the magnetic confinement and form a plasma cable with much larger density than the surroundings. He held that many of the explosive events observed in cosmic physics are produced by disruptive discharges of electric double layers in the current cables. The disruptive discharge will cause the plasma cable to pinch into a very narrow column by inward magnetic pressure of the discharge current (Krall \& Trivelpiece 1973). The resulted compressed, large-voltage and high-temperature discharge column will radiate flaring energetic photons due to ICS between the accelerated electrons and thermal photons, as sketched in Fig. [1

In an isotropic thermal emission with mean photon energy $\overline{h \nu}$, the mean energy of a scattered photon from an electron with energy $\epsilon=\gamma m c^{2}$ is $\bar{E}=\frac{4}{3} \gamma^{2} \overline{h \nu}$ (Ginzburg \& Syrovatskii 1964). For a pinch discharge column with temperature $T=10^{6} \mathrm{~K}$, the mean photon energy $\overline{h \nu} \sim 230 \mathrm{eV}$, then the mean energy of the scattered photons from electrons with $\gamma=30$ $(\epsilon \sim 15 \mathrm{MeV})$ will be $\bar{E} \sim 276 \mathrm{keV}$. For a blackbody emission of $10^{6} \mathrm{~K}$, the photon density $n \approx 2 \times 10^{19} / \mathrm{cm}^{3}$, the Compton cross-section $\sigma_{c} \approx 6 \times 10^{-25} \mathrm{~cm}^{2}$, then the electron free path $l_{c}=1 / n \sigma_{c} \approx 1 \mathrm{~km}$. A large voltage and high temperature discharge column with a length $l \gtrsim l_{c}$ should be an efficient radiator of hard X-rays and soft $\gamma$-rays.

The TGFs, intense gamma-ray flashes of atmospheric origin observed by BATSE/CGRO, are quite similar with GRBs in emission energy band and morphology. An evident correlation of TGFs with thunderstorm system (Fishman et al. 1994) and their features of energy dependence of time profiles (Feng et al. 2002) indicate that TGFs are produced by upward explosive electrical discharges at high altitude, giving an observational evidence that the discharge process can 
produce high-energy explosive events. Before a burst the energy is supposed to be stored in an electric circuit with a current $I$ and inductance $L$ as the inductive energy $W=L I^{2} / 2$. It has been realized that there is an atmospheric electrical global circuit, in which a current flows between the ionosphere and the earth with thunderstorms as a d.c. generator (see e.g. Markson \& Muir 1980). Another kind of high-energy transients similar to GRBs is the solar hard X-ray flare. Alfvén \& Carlqvist (1967) suggested exploding discharges of electric double layers to be responsible for solar flares. In their model, the energy release and particle acceleration of a flare are produced by the disruption discharge of a current of $10^{11}-10^{12} \mathrm{~A}$, flowing in a solar atmosphere circuit with a typical length $10^{9}-10^{10} \mathrm{~cm}$ and inductance $\sim 10 \mathrm{H}$.

The pinch discharge scenario could account for the energy budget even for GRBs at cosmological distances. For the typical $10^{53}$ erg isotropic-equivalent output, collimation reduces the burst energy budget to $\sim 10^{49} \mathrm{erg}$. For a circuit with a radius of stellar dimension, we can expect its inductance $L \sim 10^{2} \mathrm{H}$, then the needed current $I \sim 10^{20} \mathrm{~A}$. The required current circuit is not impossible in the astrophysical context (see e.g., Raadu 1989). Large-scale current circuits have been proposed both for the heliosphere and for the galaxy (Alfvén 1978). In the galactic case currents of $10^{17}-10^{19} \mathrm{~A}$ have been estimated (Alfvén \& Carlqvist 1978). Since the typical GRB photon energy is $\sim 100 \mathrm{keV}, \sim 10^{56}$ photons must be scattered to lead to a GRB. The needed radius $r$ and length $l$ of the discharge column to produce $N_{b}=10^{56}$ photons in $t_{\text {burst }}=30 \mathrm{~s}$ can be estimated by $2 \pi r l f t_{\text {burst }}=N_{b}$, where $f$ is the emission intensity of black body with temperature $k T(\mathrm{keV}), f=5 \times 10^{32}(k T)^{3} \mathrm{~cm}^{-2} \mathrm{~s}^{-1}$. Then the required column size $r l \sim 10^{21} /(k T)^{3} \mathrm{~cm}^{2}$. Bursts consisting of spiky components are produced by branching discharges, the size of each individual column can be much smaller.

The pinch discharge mechanism can naturally interpret many observed GRB characteristics (Li \& Wu 1997; Li 1998). Complicated morphological patterns in GRBs, such as wide variety of profile configurations, rich in fluctuation or smooth structures, rapid rise vs slower fall, weak precursor and secondary pulse, etc., are common in various electrical discharges. Producing energy spectra of smoothly-joining broken power law, so called the Band model to describe GRB spectra, is a general property of the discharge mechanism. The distribution feature of the low-energy power law slope $\alpha$ and peak energy $E_{\text {peak }}$ from the discharge model is similar to what observed in GRBs. Spectral evolution features observed in GRBs, e.g. hard-to-soft spectral evolution and time-resolved low-energy spectra over a pulse, can be reproduced by the discharge model. The typical energy dependence of GRB time profiles, the variability of hard emission is earlier and narrower than that of soft emission, is the character of a disruptive discharge mechanism as well. Here we will show in the next section that the discharge mechanism can also naturally produce strongly polarized emission.

\section{POLARIZATION}

In a pinch discharge in plasma, the electric current is along the surface of the discharge column by the skin effect. The high-temperature pinched plasma is compressed within the column. In the following calculations we suppose the local surface of a concerned pinch column can be seen as a plane, then the electric lines of force on it are parallel to each other.

Fig. 2] illustrates the ICS process of a photon with incident energy $h \nu_{i}$ and a moving electron to produce a scattered photon with energy $h \nu$, where $\psi_{i}$ and $\psi$ are the include angle of the incident and scattered photon with respect to the moving electron respectively, and the scattering angle between the incident and scattered photons $\theta=\psi_{i}-\psi$. With Lorentz transformations, the energy ratio between the incident and scattered photons in the electron rest frame (in primed notation) can be derived as

$$
\frac{h \nu_{i}^{\prime}}{h \nu^{\prime}}=1+\frac{\gamma h \nu_{i}}{m c^{2}}\left(1-\beta \cos \psi_{i}\right) \equiv \lambda\left(\psi^{\prime}, \theta^{\prime}, h \nu_{i}\right),
$$




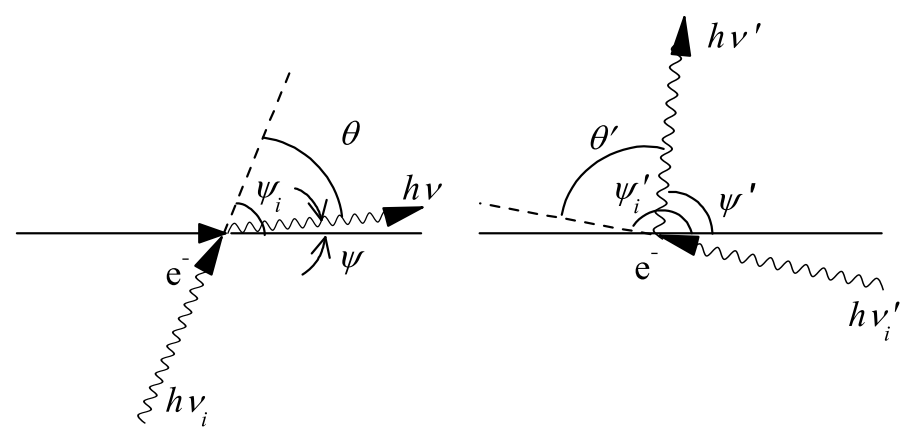

Fig. 2 Geometry for inverse Compton scattering. Left panel: in the lab system. Right panel: in the electron rest system.

where $\beta$ is the electron velocity in units of $c, \gamma=\sqrt{1-\beta^{2}}$, and $m$ the electron rest mass. We also used the relations $\psi_{i}=\arccos \left(\frac{\beta+\cos \psi_{i}^{\prime}}{1+\beta \cos \psi_{i}^{\prime}}\right)$ and $\psi_{i}^{\prime}=\theta^{\prime}+\psi^{\prime}$.

At the scattering angle $\theta^{\prime}$ the polarization $\xi$ of an ICS photon with incident energy $h \nu_{i}^{\prime}$ can be expressed as Akhiezer \& Berestetskii (1965).

$$
\xi\left(\psi^{\prime}, \theta^{\prime}, h \nu_{i}\right)=\frac{\sin ^{2} \theta^{\prime}}{\frac{h \nu_{i}^{\prime}}{h \nu^{\prime}}+\frac{h \nu^{\prime}}{h \nu_{i}^{\prime}}-\sin ^{2} \theta^{\prime}}=\frac{\sin ^{2} \theta^{\prime}}{\lambda+\lambda^{-1}-\sin ^{2} \theta^{\prime}} .
$$

The differential scattering cross-section in the electron rest system is (Klein \& Nishina 1929)

$$
\begin{aligned}
\frac{d \sigma_{T}}{d \psi^{\prime}} & \propto\left(\frac{h \nu^{\prime}}{h \nu_{i}^{\prime}}\right)^{2}\left(\frac{h \nu_{i}^{\prime}}{h \nu^{\prime}}+\frac{h \nu^{\prime}}{h \nu_{i}^{\prime}}-\sin ^{2} \theta^{\prime}\right) \sin \psi^{\prime} \\
& =\lambda^{-2}\left(\lambda+\lambda^{-1}-\sin ^{2} \theta^{\prime}\right) \sin \psi^{\prime} \\
& \equiv \mu\left(\psi^{\prime}, \theta^{\prime}, h \nu_{i}\right)
\end{aligned}
$$

For isotropic photons in the lab system, the number density of photons collided with the moving electron at a certain incident angle $\psi_{i}$ is

$$
\frac{d N}{d \psi_{i}} \propto\left(1-\beta \cos \psi_{i}\right) \sin \psi_{i} \equiv \eta\left(\psi^{\prime}, \theta^{\prime}\right) .
$$

The polarization is a Lorentz invariant, we can calculate it in the electron rest frame. From ICS of photons with energy spectrum $p\left(h \nu_{i}\right)$ in the lab system, the average polarization $\bar{\xi}(\psi)$ of scattered photons at a certain angle $\psi$ can be calculated as

$$
\bar{\xi}(\psi)=\bar{\xi}\left(\psi^{\prime}\right)=\frac{\int \xi\left(\psi^{\prime}, \theta^{\prime}, h \nu_{i}\right) \mu\left(\psi^{\prime}, \theta^{\prime}, h \nu_{i}\right) \eta\left(\psi^{\prime}, \theta^{\prime}\right) p\left(h \nu_{i}\right) d \theta^{\prime} d\left(h \nu_{i}\right)}{\int \mu\left(\psi^{\prime}, \theta^{\prime}, h \nu_{i}\right) \eta\left(\psi^{\prime}, \theta^{\prime}\right) p\left(h \nu_{i}\right) d \theta^{\prime} d\left(h \nu_{i}\right)} .
$$

We calculate the polarization at different angles $\psi$ of the observer's line-of-sight to the GRB with the discharge direction for photon energy distribution $p\left(h \nu_{i}\right)$ in the lab system being a blackbody spectrum with $k T=1,5$ and $10 \mathrm{keV}$, separately. For each temperature, the Lorentz factor $\gamma$ of moving electron is taken to be 10 and 50, respectively. As the pinched high-temperature plasma is concentrated in the discharge column, incident angles $\psi$ of photon are restricted in the range of $0-\pi$. The results are shown in Fig. [3] 


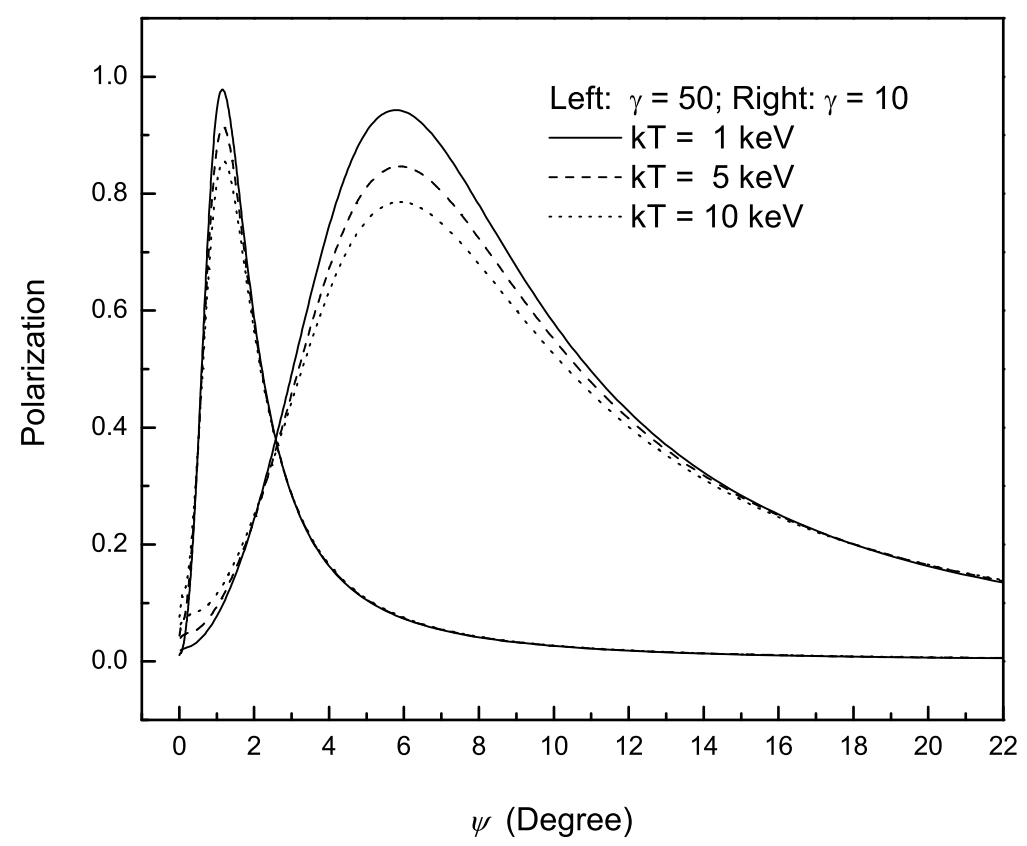

Fig. 3 Polarization vs. angle $\psi$ of observer's line-of-sight to discharge direction for electron Lorentz factor $\gamma=10,50$, and pinched plasma temperature $k T=1,5,10 \mathrm{keV}$, respectively. The calculation is for energy of scattered photons limited in $25-2500 \mathrm{keV}$.

To see the relationship of polarization to GRB photon energy expected by the discharge mechanism, we calculate the polarization for different value of $h \nu$ with given $\gamma, k T$ and $\psi$, the results are shown in Fig. 4.

From Figs. 3 and 4 we can see that a discharge column, shown in Fig. 2, can produce prompt $\gamma$-ray emission polarized in the direction perpendicular to the scattering plane, the observed amount of polarization depends on the kinetic energy of the moving electron, temperature of pinched plasma and angle between the observer's line-of-sight to the GRB and the direction of the discharge. It also depends on observed $\gamma$-ray energy, but for $h \nu \geq 500 \mathrm{keV}$ the dependency becomes weak.

\section{DISCUSSION}

During a discharge the energy released from the central engine and after it the pinched highpressure and high-temperature plasma may produce fireball and afterglow emission in the ambient medium. The geometry and environment of the discharge may influence the production of fireball and afterglow. Different from fireball shock models, the location of the GRB prompt emission in the discharge model is neither the internal shock nor external shock, but the central engine itself. In comparison with the blast-wave model, the discharge mechanism provides a more clear postulate on the nature of the GRB prompt gamma-ray emission. The GRB produc- 


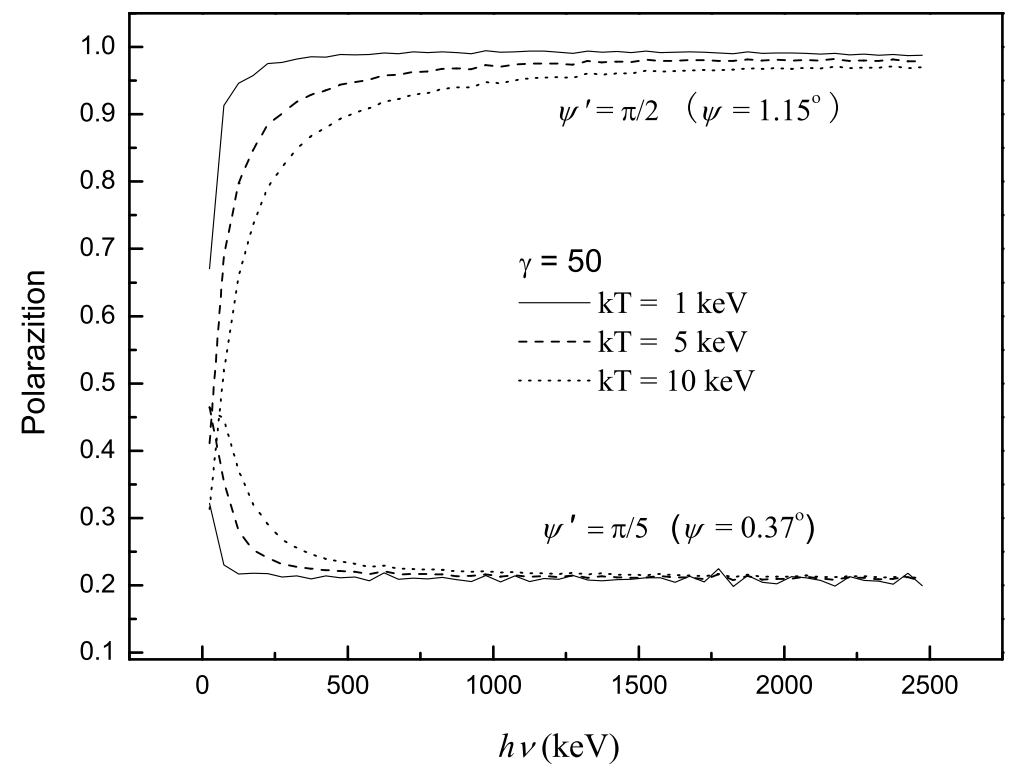

Fig. 4 Polarization vs. energy of scattered photon for electron Lorentz factor $\gamma=50$ and for different scattered angles $\left(\psi^{\prime}=\pi / 2, \pi / 5\right)$ and pinched plasma temperatures $(k T=1,5,10 \mathrm{keV})$.

ing mechanism in the scenario has no difficulty on the baryon contamination and compactness problem, and no difficulty on synchrotron "line-of-death" (Preece et al. 1998) and cooling problem (Ghisellini et al. 2000) in the synchrotron model. No need for assuming a globally organized strong magnetic field or other unusual configuration or bulk motion for fireballs, the pinch discharge itself can provide necessary conditions to generate strongly polarized $\gamma$-ray emission: the high-energy electrons and dense thermal photons for ICS and the discharge column as a preferential direction.

From Eq. (2) one can see that in the electron rest frame, at the scattered direction perpendicular to the incident one, $\theta^{\prime}=\pi / 2$, the polarization $\xi \simeq 1$ on condition that $h \nu^{\prime} \simeq h \nu_{i}^{\prime} \ll m c^{2}$. The above condition is usually satisfied by the thermal photons in a pinch discharge column. For isotropic photons in the lab frame, we calculate the number density $\frac{d N}{d \psi_{i}^{\prime}}$ at an incident angle $\psi_{i}^{\prime}$ in the electron rest frame from Eq. (44) [noted that $\psi_{i}=\arccos \left(\frac{\beta+\cos \psi_{i}^{\prime}}{1+\beta \cos \psi_{i}^{\prime}}\right)$ ] with Lorentz transformations for $\gamma=10,50$, and 100 respectively and show the result in Fig. (5). One can see that, in the electron rest frame, most photons colliding with the electron concentrate in a tapering half cone with an axis along the discharge direction. Therefore, high linear polarization can be observed at a proper observer's angle with the discharge direction.

As shown in Fig. (3), the polarization of observed GRBs depends on the angle $\psi$ between the observer's line-of-sight and the axis of the pinch column, varying between zero and unit for a fixed $\gamma$ of discharging electrons. With different observation angle one can see different polarization (from 0 to 1 ). The extreme circumstance is null polarization at the position with 


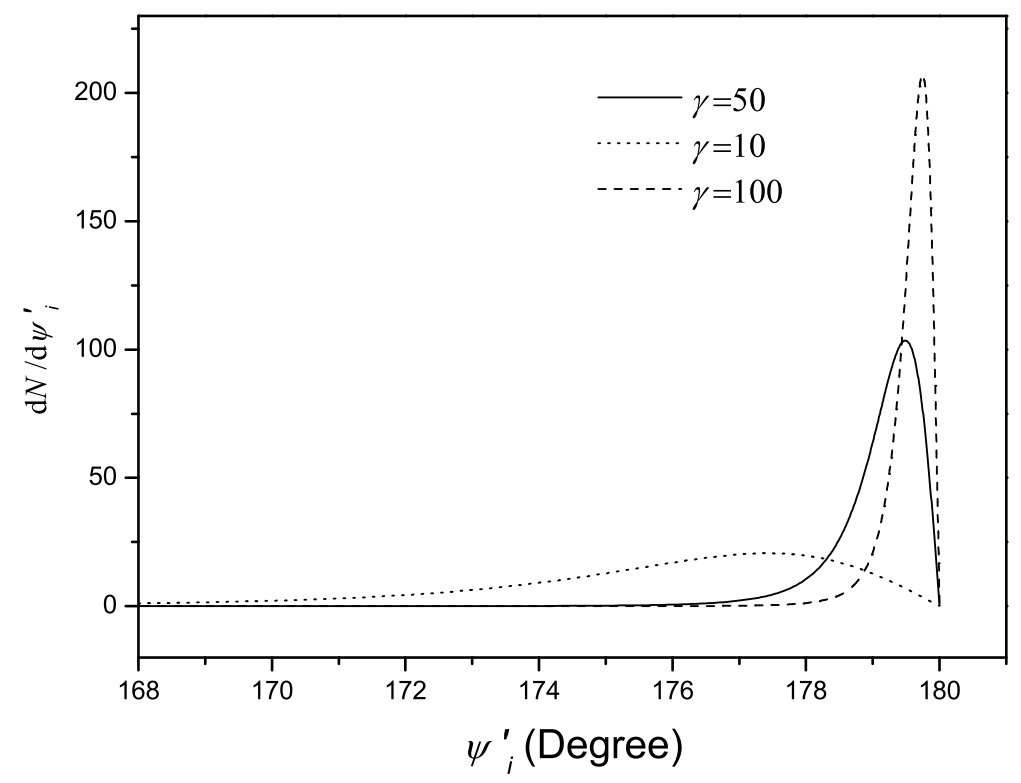

Fig. 5 Distribution of incident angles in the electron rest frame for isotropic photons in the lab frame.

$\theta^{\prime}=0$. The angle $\psi_{\max }$ where maximum polarization takes place varies with $\gamma$. Electrons moving along a discharge column are accelerated by the discharge voltage and the electron kinetic energy increases along the path. At a certain moment, the values of $\gamma$ of moving electrons should be distributed over a wide range, and hence high level of polarization can be observed over a considerable range, say several degrees, of observation angle.

Acknowledgements The referee and Dr. Lu Fangjun are thanked for helpful comments and suggestions on the manuscript. This work is supported by the Special Funds for Major State Basic Research Projects and the National Natural Science Foundation of China.

\section{References}

Alfvén H. 1978, Astrophys. Space Sci., 54, 279

Alfvén H. 1981, Cosmic Plasma, D.Reidel Publishing Company

Alfvén H. \& Carlqvist P. 1967, Solar Phys., 1, 220

Alfvén H. \& Carlqvist P. 1978, Astrophys. Space Sci., 55, 487

Akhiezer A.I. \& Berestetskii V.B. 1965, Quantum Electrodynamics, John Wiley \& Sons

Coburn W. \& Boggs S.E. 2003, Nature, 423, 415

Dar, A. \& De Rujula, A. 2003, CERN-TH/2003-137; astro-ph/0308248

Eichler, D. \& Levinson, A. 2003, ApJ, 596, L147

Feng H., Li T.P., Wu M., Zha M. \& Zhu Q.Q. 2002, Geophys. Res. Lett., 29, 6

Fishman G. J. et al. 1994, Science, 264, 1313 
Ghisellini G., Celotti A. \& Lazzati D. 2000, MNRAS, 313, L1

Ginzburg V.L. \& Syrovatskii S.I. 1964, The Origin of Cosmic Rays, Pergamon Press

Gruzinov A. 1999, ApJ, 525, L29

Klein O.\& Nishina, Zs. F. Phys. 1929, 52, 853

Krall N.E. \& Trivelpiece A.W. 1973, Principles of Plasma Physics, McGraw-Hill, Inc.

Kulsrud R.M. 1954, ApJ, 119, 386

Lazzati, D., Rossi, E., Ghisellini, G. \& Rees, M.J. 2004, MNRAS, 347, L1

Li T.P. \& Wu M. 1997, Chin. Phys. Let., 14, 557

Li T.P. 1998, in: Gamma-Ray Bursts: Huntsville, eds. C. Meegan, R. Preece, and T. Koshut, AIP Conf. Proc., 428, 825

Lutikov, M., Pariev, V. I. \& Blandford, R. 2003, AJ, 597, 998

Markson R. \& Muir M. 1980, Science, 208, 979

Medvedev M.V. \& Loeb A. 1999, ApJ, 526, 697

Preece R.D. et al. 1998, ApJ, 506, L23

Raadu M.A. 1989, Physics Reports, 178, 25

Rutledge,R.E. \& Fox, D.B. 2004, MNRAS, 350, 1272

Waxman, E. 2003, Nature, 423, 388

Wigger, W., Hajdas, W., Arzner, K., Güdel, M. \& Zehnder, A. 2004, ApJ, 613, 1088

This paper was prepared with the ChJAA LATEX macro v1.0. 